\title{
EL FILIBUSTERISMO PRECLOTURE EN EL SENADO ESTADOUNIDENSE (1789-1917)
}

\author{
ALFONSO CUENCA MIRANDA
}

(C) UNED. Revista de Derecho Politico

N.o 111, mayo-agosto 2021 
SUMARIO

I. INTRODUCCIÓN: LAS BASES CONCEPTUALES Y REGLAMENTARIAS DEL FILIBUSTERISMO CLÁSICO. II. EL FILIBUSTERISMO PRECLOTURE: PERÍODOS, MARCO PROCEDIMENTAL Y PRINCIPALES CASOS. II.1. El Senado primitivo: 1789-1820; II. 2. El filibusterismo senatorial entre 1820-1880. II. 3. El auge del filibusterismo precloture: 1880-1917. III. LA APROBACIÓN DEL CLOTURE. CONCLUSIONES. 


\title{
EL FILIBUSTERISMO PRECLOTURE EN EL SENADO ESTADOUNIDENSE (1789-1917)
}

\author{
ALFONSO CUENCA MIRANDA \\ Letrado de las Cortes Generales \\ Universidad Rey Juan Carlos ${ }^{1}$
}

\section{INTRODUCCIÓN. LAS BASES CONCEPTUALES Y REGLAMENTARIAS DEL FILIBUSTERISMO CLÁSICO}

El filibusterismo puede ser definido como la modalidad estadounidense del obstruccionismo parlamentario. En un sentido estricto el mismo se identifica con aquel obstruccionismo realizado mediante el uso prolongado de la palabra, de tal manera que el acaparamiento del turno de palabra retrasaría o impediría la aprobación de una medida. Con todo, en un sentido más amplio incluiría otras tácticas empleadas con el mismo fin: reiteradas peticiones de comprobación de quorum, quórums evanescentes, presentación de múltiples mociones y enmiendas, etc...2

1 El presente artículo forma parte, con las pertinentes adaptaciones, de la tesis actualmente en preparación del mismo autor, realizada en el marco del Programa de doctorando en Ciencias Sociales y Jurídicas de la Universidad Rey Juan Carlos. Dirección de correo web: acuencamiranda73@gmail.com. Dirección Postal: Senado. Calle Bailén nº 3. Madrid, C.P. 28071. Orcid: 0000-0002-6329-2712.

2 Para FISK y CHEMERINSKY («The Filibuster». Standford Law Review, vol. 49:181, 1997, p. 183) el filibusterismo, en términos generales, es el uso estratégico del retraso para bloquear una ley, obstruir una nominación, forzar una enmienda u obligar al Senado a adoptar cualquier otro tipo de acción ( Generally speaking, a filibuster is a estrategic use of delay to block legislation, to obstruct a nomination, to force an amendment, or to prompt other Senate action»). Por su parte, KOGER (Filibustering: a Political Historic of Obstruction in the House and Senate. The University of Chicago Press, 2010, p. 16) lo define como una conducta legislativa, o la amenaza de tal conducta, dirigida a dilatar una decisión colectiva para obtener una ganancia estratégica («a legislative behavior, or a threat of such behavior, intended to delay a colective decisión for strategic gain»). BELL (Filibustering in the U. S. Senate. Cambria Press, 2010) lo define como un esfuerzo para retrasar o impedir intencionadamente que tenga lugar una medida, nominación o actividad procedimental (an effort «to intenationally delay or prevent any measure, nomination or procedural activity from taking place»). Por su parte, BONDURANT ( tion». Harvard Journal on Legislation, vol. 48, p. 467.) mantiene un concepto estricto de filibusterismo, 
Sabido es que el filibusterismo tiene como ámbito más característico el del Senado estadounidense, si bien ni la Cámara de Representantes ni las asambleas estatales desconocen tal fenómeno, aunque en un grado mucho menor, pues, no en vano, cuentan desde hace tiempo con mecanismos que restringirían en gran medida las posibilidades del mismo $\mathrm{m}^{3}$. El Senado norteamericano es sin duda la Cámara más importante del mundo (no digamos ya entre las «segundas cámaras»), no sólo dadas sus trascendentes competencias en el arquitrabe institucional diseñado por el constituyente de Filadelfia, sino también por el relevante papel jugado por los senadores individualmente considerados. En ello es de capital importancia la libertad de palabra y su empleo obstruccionista, esto es, el filibusterismo.

El filibusterismo clásico tuvo como principal instrumento o plasmación los conocidos como discursos irrestrictos, no obstante lo cual también fueron empleados otros resortes, como ya se ha indicado, destacando entre los mismos las comprobaciones de quórum y los denominados quórums evanescentes. Por lo que respecta a su plasmación más relevante, los discursos prolongados, el Senado no contaría hasta 1917 con una Regla, la XXII, que previera el posible cierre del debate a petición de un grupo de senadores. Con todo, la adopción del cloture no supondría una disminución en el número de filibusterismos, siendo su incidencia muy marginal hasta la década de los setenta del pasado siglo. La extensión en la aprobación del cloture determinaría que el filibusterismo o, mejor dicho, el cierre del debate se transformara en una regla de votación, la exigencia de una supermayoría (60 votos en la actualidad) para la aprobación de los proyectos por el Senado estadounidense. Lo señalado explicaría que a partir de la década de los setenta del pasado siglo el filibusterismo clásico perdiera importancia, siendo la modalidad más empleada desde entonces (y aun en la actualidad) la del filibusterismo silente (stealth filibuster), articulado a través de los bolds u objeciones por parte de los senadores individuales a la tramitación de un proyecto, de tal modo que ya no es necesario agotar el uso de la palabra para bloquear un proyecto, desbloqueo que sólo se logrará cuando la mayoría consiga reunir 60 votos en apoyo de aquél.

\footnotetext{
señalando que el mismo es un abuso intencionado del privilegio de debate ilimitado («a filibuster is an intentional abuse of the privilege of unlimited debate»).

3 En la Cámara de Representantes el filibusterismo estuvo a la orden del día durante buena parte del siglo XIX (en algunos períodos con mayor intensidad que en el Senado). Dado que contaba ya desde 1841 con la posibilidad de aprobar por mayoría simple mociones de cuestión previa para el cierre del debate, la táctica principal empleada en la Cámara Baja durante la segunda mitad de la centuria fue la de los quórums evanescentes. Sería la actuación del Speaker Reed en 1890 la que pondría prácticamente fin a la misma al permitir a la presidencia el cómputo de los congresistas presentes aun cuando no respondieran al llamamiento. Por otra parte, la propia dinámica posterior de la Cámara de Representantes, por la que se articuló un férreo control del procedimiento por el grupo mayoritario, no dejo prácticamente resquicio alguno para el obstruccionismo. Véase DION, G. D. Removing the Obstructions: Minority Rights and the Politics of Procedural Change in the Nineteenth Century House of Representatives. UMI Dissertation Services, 1991,
} 
En el presente artículo se abordará el estudio del filibusterismo histórico en el Senado norteamericano, entendiendo por tal el empleado hasta la aprobación del cloture o cierre del debate en 1917, si bien el mismo perviviría hasta la década de los sesenta cuando tiene lugar su «sustitución» por el filibusterismo silente o sistema de bold (sin perjuicio que, todavía hoy, de cuando en cuando, se produzcan episodios de filibusterismo clásico o parlante).

El filibusterismo estricto, o filibusterismo parlante, tiene como presupuesto la libertad de palabra o de debate como principio rector del Senado norteamericano (así, ha sido calificado como pilar del mismo). Consagrada en la Rule XIX se establece por la misma que ningún senador podrá ser privado del uso de la palabra una vez que la haya tomado, teniendo todos ellos el derecho a usarla (solo se establece una prioridad en el reconocimiento o recognition, desde la segunda mitad del siglo pasado, en favor de los portavoces o líderes de la mayoría y minoría, pero sin que ello excluya la posibilidad de intervención del resto de senadores). Una vez obtenida del Presidente el uso de la palabra, el senador no la pierde salvo que la ceda a otro senador o infrinja una norma de decoro de la Cámara (entendida esta como nuestra disciplina parlamentaria). Con todo, con el tiempo se fueron estableciendo algunas limitaciones no sustanciales, destacando entre ellas la prohibición de que un senador pueda hacer uso de la palabra más de una vez sobre un mismo asunto en la misma sesión, salvo autorización de la propia Cámara.

Como se ha indicado, el otro gran mecanismo obstruccionista en la Cámara Alta estadounidense, con el que el filibusterismo parlante ha guardado históricamente una estrecha relación, es el relacionado con el quórum. La Constitución de 1787 (art. 1, secc. $\mathrm{V}$, parágrafo $1^{\circ}$ ) establece que para la conducción de sus asuntos deberán estar presentes la mitad de los miembros de la Cámara respectiva, señalando que en cualquier momento podrá demandarse por un senador la comprobación de tal extremo (posibilidad desarrollada en los Reglamentos de Cámara y Senado). Así, en el constitucionalismo histórico estadounidense se acudió a las múltiples peticiones de comprobación de quórum como medio para retrasar la aprobación de medidas en tramitación. En este punto, ha de tenerse en cuenta que, en particular en el Senado, la comprobación del quórum llevaba aparejada la consunción de un tiempo extenso, toda vez que ha sido costumbre inveterada en la Cámara (y aún lo es) que a los debates asistan muy pocos senadores (a veces solo el interviniente), por lo que cuando se les llama a comprobación de quórum deben acudir desde otras dependencias, dejándose un lapso amplio (quince minutos y en ocasiones más) para que se lleve a cabo tal comprobación ${ }^{4}$.

Por lo que respecta a la relación entre el filibusterismo parlante y el practicado a través de las comprobaciones de quórums, ha de señalarse que, históricamente, la mayoría empleó como contramedida de aquél el mantener al Senado en sesión continua («round the clock sessions»), de modo que el filibusterista se viera obligado a inter-

4 BETH, R. S. et HEITSHUSEN, V. Filibuster and Cloture in the Senate. Congressional Research Service, december 2014 .

(C) UNED. Revista de Derecho Politico

N. ${ }^{\circ} 111$, mayo-agosto 2021, págs. 137-162 
venir ininterrumpidamente, poniendo a prueba, por tanto, su resistencia física. Sin embargo, los senadores filibusteristas contaban con un antídoto, en este caso, la posibilidad de que, previa petición de la comprobación de quórum, se acreditase que este no se reunía, lo que obliga, de acuerdo con las normas del Senado, a concluir (adjourn) la sesión hasta el día siguiente. Ello conllevaba retrasar adicionalmente la consideración de la medida, pues, al tratarse de un nuevo día legislativo, se abordan en primer lugar otros asuntos, en concreto, los que configuran el denominado «morning hour». De este modo, se obligaba a la mayoría a permanecer en la Cámara o sus aledaños si quería persistir en su guerra de desgaste o de resistencia contra la minoría filibusterista. De ahí que no hayan sido escasas las ocasiones en las que se asistiera a la escena de senadores acampados dormitando en las salas vecinas al pleno.

\section{EL FILIBUSTERISMO CLÁSICO PRECLOTURE: PERÍODOS, MARCO PROCEDIMENTAL Y PRINCIPALES CASOS}

En el análisis del filibusterismo clásico precloture pueden establecerse diferentes períodos (que discurren, no por casualidad, de manera cuasi-paralela a los sucesivos sistemas de partidos en Estados Unidos) en función de su diferente desarrollo y a la temática anudada a los mismos. Así, cabría distinguir: 1) Una primera etapa, la del Senado primitivo, que abarcaría los años 1790 a 1820, en la que se asiste a fenómenos episódicos de filibusterismo, en correspondencia con la escasa relevancia que por entonces tuviera la Cámara Alta en el sistema político; 2) Una segunda etapa, que abarcaría los años 1820 a 1880, coincidente con la conformación del Senado como asamblea dominante en el escenario estadounidense, en la que, en el marco del conflicto de la esclavitud y su correspondencia con la expansión territorial de la Unión, se recurriría con mayor frecuencia al filibusterismo como arma táctica; 3) El tercer período analizado comprendería los años 1880 a 1917, pudiendo calificarse de edad de oro del filibusterismo clásico en Estados Unidos, siendo el mismo empleado profusamente en el contexto del enfrentamiento político entre republicanos y demócratas y, en especial, por los senadores integrantes del conocido como movimiento progresista.

II. 1. El Senado primitivo: 1789-1820

a) Factores generales con influencia en el filibusterismo

En el Senado primitivo difícilmente puede hablarse de filibusterismo en sentido propio. Se trataba de una Cámara de muy reducidas dimensiones y con una significación política muy capitidisminuida en comparación con su alter ego en Capitol Hill. 
Así, el número de sesiones y la duración de los períodos ordinarios de las mismas serán muy limitados, especialmente, en comparación con las ratios que se darán en la evolución posterior del Senado (la propia duración de las sesiones era breve, desde el mediodía hasta las tres de la tarde, los martes y miércoles). En relación con ello, el número de iniciativas tramitadas tampoco fue muy significativo 5 . El hecho de que existiera una presión muy escasa de trabajo y, por tanto, de sacar adelante una importante carga de proyectos e iniciativas, provocaría que, en caso de haberse producido, los costes del filibusterismo hubiesen sido muy reducidos para la propia Cámara. En segundo lugar, ha de recordarse que las sesiones de este primer Senado no fueron públicas hasta $1795^{6}$, no existiendo por otra parte un diario de sesiones como tal. En general (aunque debe admitirse que también podría haber influido en sentido contrario), la no publicidad inicial de las sesiones, así como el escaso interés que suscitaba en la prensa el escenario senatorial en comparación con la Cámara de Representantes, harían que el filibusterismo no actuase como potencial medio de atracción de la opinión pública o del electorado del Estado al que perteneciera el senador o senadores obstruccionistas.

Por otra parte, la composición reducida del Senado (especialmente en comparación con la Cámara de Representantes) daba a la dinámica de la Cámara Alta unas notas de intimidad y compañerismo que casaban mal con un indebido uso de la palabra con fines obstruccionistas. Este factor, que, como se verá, ha estado siempre presente en la cultura institucional del Senado norteamericano, operaba, por razones obvias, con mayor intensidad en los primeros años de funcionamiento del mismo. A ello debe añadirse la propia consideración del Senado como una Cámara de especial dignidad, modelada en parte, por lo que se refiere a cuestiones procedimentales y formales, a semejanza de la Cámara de los Lores británica ${ }^{7}$. Esa particular solemnidad y cortesía fue interiorizada por los senadores desde el primer momento a la hora de conducir los debates, constituyendo un signo de identidad del Senado en contraste con la bulliciosa y apasionada Cámara Baja, cuyos debates estaban plagados de incidentes y agrias disputas ${ }^{8.9}$.

5 En este sentido, hasta 1812 el número de proyectos iniciados en la Cámara baja triplicaba el de los presentados en el Senado. Asimismo, entre 1789 y 1814 la media del número de votaciones por Congreso (o Legislatura) sobre iniciativas legislativas fue de 160 en la Cámara de Representantes frente a 130 en el Senado. BINDER, S. A. et SMITH, S. S. Politics or Principle? Filibustering in the United States Senate. Brookings Institution Press, 1997, pp. 40-42.

6 Incluso con posterioridad a dicha fecha las sesiones en las que se tramitaban tratados internacionales y nominaciones de cargos públicos siguieron siendo a puerta cerrada.

7 SWIFT, E. K. The Making of an American Senate, Reconstitutive Change in Congress, 1787-1841. University of Michigan Press, 1996.

8 MACNEIL, N. et BAKER, R. A. The American Senate: an insider's history. Oxford University Press, 2013, pp. 303-305.

9 Finalmente, las propias condiciones físicas y ambientales del Senado podrían citarse como factor disuasorio o, al menos, dificultativo, del filibusterismo. No hay que olvidar que la Cámara en este perío-

N. ${ }^{\circ} 111$, mayo-agosto 2021, págs. 137-162 
b) Marco procedimental

Ha de subrayarse como factor más importante a tener en cuenta en este primer período de la singladura senatorial, la existencia hasta 1806 de la denominada moción de cuestión previa, moción que en teoría se correspondería con lo que conocemos como moción de cierre $^{10}$. Y decimos que en teoría porque existe un intenso debate acerca de la auténtica virtualidad de la referida moción, dividiéndose la doctrina entre aquellos autores que señalan que la misma operaba como moción de cierre frente a aquellos que sostienen que más bien fue empleada con fines opuestos, esto es, precisamente, obstruccionistas ${ }^{11}$. Ello es así por cuanto que el rechazo de la moción suponía el aparcar el debate de fondo hasta la siguiente sesión. En cualquier caso, la reforma de las Rules aprobada en 1806 eliminó tal moción (que subsistió y subsiste aun en la Cámara de Representantes), no estando claros los motivos subyacentes a ello.

De otra parte, ha de constatarse que estos primeros años de andadura senatorial rigió de manera convencional lo señalado en el Manual de Jefferson, en el sentido de que se prohibían las alocuciones impertinentes o completamente ajenas a la cuestión debatida (regla que, como se verá, fue abandonada posteriormente en el Senado).

\section{c) Principales casos}

Como ya se ha indicado, en este período difícilmente puede hablarse de filibusterismo en sentido estricto, no faltando, empero, algunos episodios esporádicos. El primero de ellos se produjo en 1790 a propósito del debate habido en relación con el posible traslado de la sede del Senado a Filadelfia. En este caso, senadores de Estados del Sur (principalmente, Virginia y las dos Carolinas) se concertaron para impedir la aprobación de la propuesta empleando diversas tácticas, entre ellas intervenciones prolongadas, hasta que llegara un senador ausente por enfermedad que decantaría en su favor las votaciones (el senador fue trasladado en camilla a la Cámara) ${ }^{12}$. En 1798,

do era de pequeñas dimensiones, desarrollándose las sesiones en un ambiente no agradable, principalmente debido al asfixiante calor húmedo de Washington en verano y al frío riguroso de los inviernos.

10 La norma tenía como precedente lejano la homónima moción introducida en el Parlamento británico en 1604, siendo adoptada por el Segundo Congreso Continental al aprobar su Reglamento en mayo de 1778. Con dichos antecedentes, fue introducida por el Senado y por la Cámara de Representantes al aprobar sus Reglamentos, continuando en esta última Cámara hasta nuestros días. Véase GOLD M. B. et GUPTA, D. «The constitutional option to change Senate rules and procedures: majoritarian means to overcome the filibuster». Harvard Journal of Law E Public Policy, vol. 28, 2004, pp. 214-215.

11 FISK, C. et CHEMERINSKY, E. «The Filibuster»... op. cit., p. 188. Entre los autores que señalan que la cuestión previa en el Senado primitivo no operaba como mecanismo de cierre del debate, COOPER, J. Previous Question: It's Standing as Precedent for Cloture in Senate, Senate Document No. 87-104, 87 $7^{\text {th }}$ Congress, 2nd Session (Washington: U.S. Government Printing Office, Volume 2, Serial $12445,1962), 26$. En contra de dicha posición, calificando a la moción de cuestión previa como instrumento que ponía fin al debate, entre otros GOLD et GUPTA (The Constitucional option..., op. cit. p. 213).

12 Véase FISK et CHEMERINSKI. «The Filibuster»... op. cit. pp. 187-8. 
en el marco del enfrentamiento entre federalistas y demócratas-republicanos a propósito de la política a seguir en relación con la Francia revolucionaria y la antigua metrópoli, se asiste a otro episodio obstruccionista ${ }^{13}$. Ante la exigencia de la publicación de las instrucciones dadas por el gobierno federalista a los negociadores diplomáticos con Francia en el conocido como asunto XYX, senadores afines al ejecutivo acudieron a la moción de cuestión previa con el fin de que, si era rechazada, se retrasase la aprobación de acuerdo alguno, si bien la moción fue finalmente aprobada.

\section{2. El filibusterismo senatorial entre 1820 y 1880}

a) Factores generales con incidencia en el filibusterismo

En esta etapa el filibusterismo hace su auténtica aparición. Una serie de factores generales inciden en ello. En primer término, ha de subrayarse que el Senado alcanza en esta época la que ha sido considerada por muchos como su edad dorada, con figuras excepcionales que destacan por su talla política y virtudes oratorias ${ }^{14}$. El peso de la Cámara se incrementa exponencialmente como consecuencia en gran parte del aumento en su tamaño y de las cuestiones surgidas al hilo de la expansión territorial hacia el Oeste.

El mayor protagonismo de la Cámara va acompañado, como es lógico, de la mayor relevancia de los senadores. En relación con ello, en este período comienza a producirse un desligamiento respecto a las Asambleas estatales que los designan (en la década de 1840 desaparecen las anteriores instrucciones vigentes en algunos Estados). Paralelamente, los senadores van conformando sus propias bases partidarias, gracias en gran parte a los nuevos medios de transporte y, sobre todo, a los de comunicación, que permiten dar una mayor publicidad a su acción senatorial.

De otra parte, se asiste a una creciente polarización política. En el plano inclinado que finalmente conducirá a la contienda civil, las posiciones políticas se radicalizan, con una fuerte división entre los dos grandes partidos y, especialmente, entre territorios. En consecuencia, el debate se agría y la tentación de recurrir a cualesquiera medios para derrotar las propuestas del adversario y sacar adelante las propias explicará el recurso al filibusterismo ${ }^{15}$.

Tres factores adicionales cabe señalar. En primer lugar, el incremento sustancial de la agenda legislativa del Senado ${ }^{16}$ hace más dañinos los efectos de la obstrucción, al impedir la aprobación no sólo de la medida frente a la que se dirige, sino de otros proyectos en lista de espera, siendo particularmente «efectivos» en este sentido los

\footnotetext{
13 Véase COOPER, J. Previous Question..., op. cit. p. 18.

14 En este sentido se suele hablar del «trío mágico» formado por Clay, Webster y Calhoun.

15 MACNEIL et BAKER. The American Senate... op. cit. pp. 285-7.

16 El número de leyes aprobadas se duplicó entre 1800 y 1840, doblándose de nuevo entre este año y 1870 .
}

(C) UNED. Revista de Derecho Politico

N. ${ }^{\circ} 111$, mayo-agosto 2021, págs. 137-162 
filibusterismos planteados en los días finales de los períodos de sesiones, en especial, de las conocidas como «short sessions» ${ }^{17}$. Por otro lado, el incremento en el número de senadores hace que los primitivos elementos de autocontención ya no rijan con su original intensidad ${ }^{18}$. Difícilmente puede hablarse ya de «un grupo de hombres junto al fuego». Por último, las mejoras en las condiciones físicas imperantes en la Cámara favorecerán la exposición de argumentos durante horas.

\section{b) Marco procedimental}

En este período se producen los primeros debates acerca de la existencia de un derecho irrestricto de uso de la palabra. Así, en 1841, con ocasión de sendos filibusterismos, se planteó por vez primera limitar el tiempo de las intervenciones. Clay propuso establecer el límite de una hora por intervención, y, ante el rechazo inicial de la misma por su propio caucus, la reintroducción de la cuestión previa, inadmitiéndose también. Dos años más tarde se reprodujo el intento por parte del mismo senador, con idéntico resultado. En ambos casos, la principal oposición a la articulación de límites en los debates procedió del grupo sureño (Calhoun, en un caso, y Jefferson Davies, en el otro, fueron los principales detractores de la medida) quedando identificada desde entonces la libertad de palabra con los defensores de los derechos de los Estados y la minoría ${ }^{19}$.

En conexión con lo anterior, ha de señalarse que la libertad de palabra en un sentido material quedó definitivamente establecida en el Senado en 1856 cuando una propuesta de resolución de modificación de las Rules, por la que se disponía que todo senador debía ceñirse a la cuestión objeto de debate, fue rechazada ${ }^{20}$. El principio quedaría reforzado en 1876 al permitirse a un senador leer un documento completamente irrelevante y ajeno a la cuestión principal debatida.

Por otra parte, se introduce en esta etapa la práctica obstruccionista de los vanishing quorums o quórums evanescentes. Por la misma, los senadores obstruccionistas permanecían callados y no eran computados a efectos de votación. Tras la pertinente comprobación de la ausencia de quórum y la sustanciación del llamamiento a tales efectos, los senadores filibusteristas respondían afirmativamente, y, acto seguido, al

17 Así, cabe recordar que, con anterioridad a la aprobación en 1933 de la XX Enmienda a la Constitución, cada dos años el período de sesiones (sessions) comenzado en diciembre terminaba el 4 de marzo, no reuniéndose la Cámara de nuevo hasta pasados ocho meses (salvo convocatoria de sesión especial por el Presidente federal). WAWRO y SCHICKLER (Filibuster: obstruction and lawmaking in the U. S. Senate. Princeton University Press, 2006, pp. 76-87) señalan el filibusterismo contra el Force Bill como un ejemplo paradigmático de intensidad asimétrica: los demócratas querían el rechazo del proyecto más de lo que los republicanos deseaban su aprobación.

18 La Cámara pasará de 46 miembros en el $16^{\circ}$ Congreso (1819-1821) a 76 en el 46 (1879-1881).

19 Véase BINDER, S. A. et SMITH, S. S. Politics or Principle?..., op. cit., pp. 55-57.

20 Véase BEEMAN, R. R. «Unlimited Debate in the Senate: The First Phase», 83 Political Science Quaterly, 1968, pp. 419-421. 
repetirse la votación, volvían a permanecer callados, lo que reproducía el proceso. Esta táctica, procedente de la Cámara Baja, se importaría por el Senado en 1851, en donde subsistiría durante más de medio siglo.

Ya en este período se producen los primeros intentos de poner coto a los episodios filibusteristas, si bien con efectos limitados. Así, se articularían los célebres acuerdos de consentimiento unánime (unanimous consent agreements), figura capital en el posterior devenir de la Cámara (y aun en el actual). Por medio de los mismos se flexibiliza un procedimiento que, de seguir al pie de la letra lo establecido en las Rules, sería excesivamente prolijo y encorsetado. Algunos de los referidos acuerdos establecen la fijación por unanimidad de un calendario de tramitación de una iniciativa, con una fecha tope para la finalización del debate y la consiguiente votación. De otro lado, cabe destacar la aprobación en 1872 de la denominada Anthony Rule por la que se anteponen en el calendario legislativo las iniciativas incontrovertidas a aquellas que sí suscitaban oposición. De este modo, aquéllas se tramitaban tras el morning bussiness de cada sesión, no pudiendo ningún senador intervenir por más de cinco minutos (dicha norma sigue vigente, constituyendo la actual Regla VIII del Senado). Finalmente, cabe mencionar que en este período se suscitarán algunos intentos de reintroducir la moción de cuestión previa, siendo todos ellos rechazados por el Pleno (así, los ya señalados casos de 1841 y 1843 y, posteriormente, en $1850,1862,1869,1870$ y 1873$)^{21}$.

c) Supuestos: caracteres generales y casos destacados

En el período reseñado aparecen los primeros filibusterismos dignos de ser calificados como tales, si bien el fenómeno no alcanzará aun cifras «preocupantes», variando los cálculos entre diez y doce filibusterismos en los sesenta años analizados. Destaca sobre todo la vinculación clara de los mismos con el enfrentamiento político-territorial que conduciría a la guerra civil. Así, será con ocasión de la expansión de la Unión hacia el Oeste y de la problemática respecto al estatuto esclavista o abolicionista de los nuevos Estados cuando se produzcan los episodios más estridentes de filibusterismo.

Con todo, los primeros casos se inscriben más bien en el contexto (con resonancias territoriales, en cualquier caso) del antagonismo político entre demócratas y whigs, una vez concluida la denominada «era de los buenos sentimientos», marcada por la hegemonía prácticamente absoluta de los demócratas-republicanos ${ }^{22}$. En dicho marco, la valoración de la figura del expresidente Jackson dará pie en 1837 al que se ha

21 BYRD, R. C. The Senate 1789-1989: Addresses on the history of the United States Senate, 1991, pp. $115-118$.

22 Véase DANGERFIELD, G. The Era of Good Feelings. Harcourt, Braced \& World, Inc. New York \& Burlingame, 1952.

(C) UNED. Revista de Derecho Politico

N. ${ }^{\circ} 111$, mayo-agosto 2021, págs. 137-162 
considerado como la inauguración del filibusterismo ${ }^{23}$, cuando la nueva mayoría demócrata presentó una propuesta para eliminar de un Journal de 1834 la censura de aquel aprobada por la Cámara. Ante los discursos prolongados de los whigs, los senadores de la mayoría se pertrecharon con viandas dispuestos a mantener al Senado en sesión continua con el fin de agotar a la oposición. Finalmente, esta claudicó, si bien se produjeron altercados cuando «gráficamente» se procedió a romper las hojas del Journal donde se contenía la censura. En 1841 tuvieron lugar dos nuevos episodios $^{24}$. El primero se refería a una cuestión interna o administrativa de la Cámara, como era la contratación de los impresores oficiales, realizada en el período que mediaba antes de la inauguración de la nueva legislatura, con una mayoría que iba a cambiar a resultas de las elecciones. La nueva mayoría whig propuso el despido de los referidos oficiales encontrando una gran resistencia articulada mediante discursos prolongados que retrasaron seis días la aprobación definitiva de la medida ${ }^{25}$. El segundo caso producido en dicho año destaca por cuanto que se trató del primer filibusterismo en el que un grupo de senadores confesaron abiertamente el empleo de tácticas dilatorias con el fin de impedir la aprobación de una medida. El obstruccionismo tuvo lugar frente al proyecto de creación del segundo Banco Federal, articulándose de nuevo mediante largas intervenciones por parte de senadores demócratas, lo que consiguió dilatar un mes la votación correspondiente. En esta ocasión el filibusterismo fue parcialmente exitoso, pues se alcanzó finalmente un compromiso entre mayoría y minoría.

En los años siguientes los filibusterismos presentados se insertan plenamente en el debate territorial entre abolicionistas y esclavistas al hilo de la admisión de nuevos Estados. No fue el caso, empero, del primero de ellos, a raíz de la propuesta de incorporación de Oregón en 1846, en el que la oposición a la admisión derivaba principalmente del temor ante un posible enfrentamiento con la antigua metrópoli con ocasión de la misma. Con todo, tras un retraso de más de dos meses a consecuencia del filibusterismo parlante, la propuesta fue aprobada ${ }^{26}$. Meses más tarde se suscitaría

23 BURDETTE, en su obra ya clásica sobre el filibusterismo histórico en el Senado (BURDETTE, F. Filibustering in the Senate, Princeton University Press, 1940, pp. 15-19), arranca su exposición casuística de los episodios filibusteristas en dicha fecha, si bien señala también como precedentes aislados las intervenciones prolongadas protagonizadas por el senador Randolph en los años anteriores. Por su parte, KOGER (Filibustering... op. cit. p. 62) data el primer episodio filibusterista en el Senado en 1831, a propósito de la tramitación de un proyecto de construcción de un puente levadizo sobre el Potomac, que dio lugar a la presentación de múltiples mociones dilatorias.

24 Precisamente, no faltan autores que sitúan en 1841 el comienzo del filibusterismo senatorial, al estimar que fue en dicho año cuando por vez primera se utiliza de modo significativo la táctica dilatoria de las intervenciones prolongadas (FISK et CHEMERINSKY, «The Filibuster» ... op. cit. p. 191).

25 Véase KOGER, G. (Filibustering... op. cit. p.63), quien señala que, además, se recurrió a la táctica de los quórums evanescentes.

26 Se había aprobado por vez primera mediante consentimiento unánime la fijación de una fecha límite para el sometimiento a votación del proyecto. FISK et CHEMERINSKY, «The Filibuster», op. cit. pp. 194-195. 
otro episodio obstruccionista a propósito del conocido como Wilmot Proviso (o Compromiso Wilmot) que intentó introducirse vía enmienda en un proyecto de fondos para la guerra con México. Mediante aquél se pretendía establecer que la esclavitud nunca sería introducida en los territorios que en el futuro se incorporaran a la Unión pertenecientes con anterioridad a México. Las tácticas dilatorias protagonizadas por senadores de Estados del Sur provocarían que la enmienda y el proyecto no fueran sometidos a votación, ante la finalización del período de sesiones. En el siguiente se reeditaría un intento con idéntico resultado (en cuanto a la mención de la esclavitud, no así a la aprobación de fondos, en este caso, para comprar territorios pertenecientes al vecino meridional).

En 1850 y 1856 se reproducirán escenas obstruccionistas a propósito de la admisión de nuevos Estados, California y Kansas, respectivamente, y de la prohibición o tolerancia en los mismos de la esclavitud. En el primer caso el filibusterismo no fue exitoso, admitiéndose al nuevo Estado, mientras que en el segundo sí (Kansas no sería admitida en la Unión hasta 1861, en los inicios de la contienda civil). Se empleó una amplia panoplia de elementos obstruccionistas (en especial en el segundo): intervenciones prolongadas, presentación de múltiples mociones de suspensión (adjournment), de posposición de la iniciativa (lay on the table), de comprobaciones de quórum, etc... Particularmente virulento fue el debate en relación con la admisión de Kansas, en el que incluso un senador llegó a agredir a otro con una barra del vestíbulo, hiriéndole de gravedad (le golpeó reiteradamente en la cabeza), dejándole secuelas de por vida ${ }^{27}$.

En plena guerra civil tuvo lugar un intenso episodio obstruccionista, con la utilización de múltiples tácticas y una organización muy lograda (se establecieron turnos de relevos para que los filibusteristas pudieran tomar aliento). Así, en 1863 la presentación del correspondiente bill de indemnidad por el que se exoneraba al presidente Lincoln de cualquier responsabilidad por la suspensión del habeas corpus dio pie a una fuerte resistencia por parte de un sector de la Cámara ${ }^{28}$. El filibusterismo se planteó respecto al informe de la conferencia intercameral que conciliaba las diferencias entre los textos aprobados por ambas Cámaras. Particularmente tensa fue la sesión del 27 de enero de 1863 cuando un senador fue expulsado del hemiciclo por referirse en términos injuriosos al Presidente federal, tras lo que el mismo reapareció en el Pleno portando un arma y amenazando al Sargento de Armas. En los días siguientes se sucedieron discursos maratonianos con relevos y la presentación de múltiples mociones de suspensión. La derrota de los filibusteristas se produjo gracias a un episodio de picaresca, cuando no de arbitrariedad. En un momento en que había asumido la presidencia pro tempore el senador republicano Pomeroy, éste indicó en voz apenas audible que se sometía inmediatamente a votación el informe, añadiendo la fórmula clásica «aquellos en favor del mismo digan sí, aquellos opuestos no» y concluyendo

27 Véase BURDETTE, F. Filibustering in the Senate..., op. cit., pp. 28-30.

28 Véase KOGER, G. Filibustering... op. cit. pp. 69-70. 
«este es el voto, el informe es aprobado». Muchos senadores no se dieron cuenta de lo que había pasado. Cuando, transcurridos unos minutos, la oposición fue consciente de la situación, presentó apelación frente a la decisión presidencial, algo a lo que el Presidente se negó (de forma contraria a las Rules). Reunido el Senado al día siguiente, las protestas continuaron, exigiendo los obstruccionistas que el informe que ya se había remitido a la Cámara Baja volviera a aquél. La solicitud fue rechazada en votación plenaria.

Tras la guerra, la conocida como Reconstrucción fue objeto de tácticas filibusteristas con el fin de impedir la aprobación de proyectos relacionados con aquélla. Así, en 1865 la readmisión de Luisiana fue ocasión de un filibusterismo cuya particularidad es que aunó (aunque por motivos opuestos) a senadores republicanos y demócratas, quienes usaron abusivamente de la palabra y recurrieron a los quorum evanescentes. Siendo inminente el fin del período de sesiones, la propuesta fue retirada. En segundo término, ya en 1879 sería la tramitación de una propuesta por la que la mayoría demócrata pretendía derogar la presencia de tropas federales en las elecciones estatales en el Sur la que fuera obstruida por la minoría republicana. Como hecho novedoso, la obstrucción descansó casi exclusivamente en mociones dilatorias, votos por llamamiento y rupturas de quorum. Respecto a estas últimas, los republicanos, sentados en sus escaños se negaron a dar sus nombres en el llamamiento, forzando al Sargento de Armas a traer al hemiciclo a los senadores ausentes. La sesión del 18 de junio se prolongó toda la noche y se levantó a mediodía, reanudándose diez minutos más tarde. En dicho momento un senador solicitó la lectura del Journal de la sesión anterior. Como éste, por razones obvias, no había podido ser completado, se retrasó cualquier avance, pues el Presidente recordó que entretanto no podía ser aprobada ninguna medida. Se apeló tal decisión, lo que dio lugar a su vez a la presentación de una moción de posposición de la apelación, y, ante la falta de quorum, pues los senadores se negaron a responder, la apelación no prosperó. Se inició entonces una discusión sobre las facultades presidenciales en la comprobación del quorum, adoptándose la decisión de que se podía contabilizar a los senadores presentes, aunque no respondieran al llamamiento a los efectos del quorum de presencia, pero no del de votación ${ }^{29}$. Ante el bloqueo «imperante», el Senado levantó la sesión. Tras una nueva jornada de discursos prolongados, la iniciativa fue finalmente aprobada en la madrugada del 21 de junio.

\section{3. El auge del filibusterismo precloture: 1880-1917}

El período comprendido entre 1880 y 1917 va a suponer un incremento exponencial de los filibusterismos senatoriales, amenazando con bloquear la adopción de importantes decisiones en un país camino de convertirse en la primera potencia mundial.

29 Véase BURDETTE, F. Filibustering, op. cit. pp. 35-39. 
a) Factores

Destaca especialmente entre los factores que influyeron en el fenómeno comentado el aumento en el propio tamaño del Senado en cerca de un $30 \%$, que en el período analizado pasa de 76 senadores a 96 (en 1915). Tal hecho tuvo importantes repercusiones que influyeron en el auge del filibusterismo: desde la atenuación de las vinculaciones interpersonales entre senadores que había jugado como elemento de autorestricción frente a aquél, hasta sus mayores posibilidades dado el mayor número de intervinientes en los debates ${ }^{30}$. El desarrollo de los medios de transporte ahondaría también en la atenuación de los vínculos señalados, toda vez que permitía a los senadores pasar más tiempo en sus Estados de origen, lo que también contribuiría a reforzar los lazos entre éstos y sus electores ${ }^{31}$.

En consonancia con el aumento del tamaño del Senado, y motivado también por la acelerada industrialización y desarrollo del país, se aprecia un incremento considerable de la carga de trabajo de la Cámara, lo que hará más dañino el potencial del filibusterismo. En paralelo, en el período señalado, se asiste a la conformación de la moderna Presidencia (ya cuasi imperial), con un reforzamiento de su figura (motivado por el perfil de varios de sus ocupantes), lo que se traducirá, a los efectos que nos interesan, en la «inmisión» de la Casa Blanca en el proceso legislativo. Por vez primera la Presidencia se va a configurar como una parte activa en la agenda legislativa, lo que provocará mayores tensiones entre el ejecutivo y el legislativo, influyendo decisivamente en el considerable aumento de los episodios filibusteristas ${ }^{32}$.

Por otra parte, los partidos políticos se convierten ya definitivamente en este período en los principales protagonistas de la vida pública norteamericana. Las maquinarias electorales alcanzan un grado de desarrollo del que habían carecido hasta el momento, incrementándose las necesidades de financiación. El mayor peso de los partidos se dejará sentir en la dinámica interna de las Cámaras. Ello se evidencia primeramente en lo que respecta al partido republicano, y, de este modo, en el Senado se asiste al dominio en una gran parte del período de la conocida como «banda de los cuatro» (llamada así por comprender a los líderes de dicho partido en la Cámara Alta). Por parte demócrata se conforma también la hegemonía (más tarde trasladada al conjunto de la Cámara) del grupo sureño, de particular relevancia en lo que concierne al filibusterismo. El dominio absoluto del Sur por los senadores demócratas hacía que los mismos, en virtud del principio de la seniority, ocuparan con frecuencia la presidencia de las Comisiones de las Cámaras, incluidas las más relevantes, lo que

30 Tal es la tesis sostenida por WAWRO, G. J. et SCHICKLER, E. (Filibuster..., op. cit., p. 188), quienes añaden como factor explicativo del aumento del obstruccionismo el hecho de que a principios del siglo XX se produjera un aumento considerable en el número de senadores novatos, lo que contribuiría a que las convenciones tradicionales del Senado en relación con el uso de la palabra y el obstruccionismo jugaran un menor papel como posible antídoto frente al filibusterismo.

31 Así, KOGER, G. Filibustering, op. cit, p. 84.

32 Véase MACNEIL, N et BAKER, R. A. The American Senate... op. cit. pp. 73-95.

(C) UNED. Revista de Derecho Politico

N. ${ }^{\circ} 111$, mayo-agosto 2021, págs. 137-162 
jugará como elemento inhibidor para el resto en lo que se refiere a su posible resistencia u oposición frente al obstruccionismo protagonizado por aquéllos o en lo que concierne a la aprobación de eventuales medidas de reforma frente a la libertad absoluta de debate.

También desde el punto de vista partidista, ha de subrayarse especialmente la irrupción en el período del movimiento populista-progresista. El mismo presentaba un carácter transversal en términos ideológicos (si bien sus parlamentarios y bases electorales originales procedían en su mayor parte de la órbita republicana), preconizando una transformación del sistema de cara a conectar con los verdaderos intereses del pueblo norteamericano. Este movimiento habría de tener una honda repercusión en la vida política y, por ende, en la parlamentaria nacional con la llegada a las Cámaras de auténticos mavericks de la política (ajenos a cualquier disciplina de partido) que no dudarán en emplear el filibusterismo para lograr sacar adelante las reformas que consideran inaplazables (como instrumento de presión al bloquear otras iniciativas). Asimismo, ha de tenerse en cuenta, al estar íntimamente con la virtualidad del filibusterismo, que los senadores progresistas tendrán su base electoral en los nuevos Estados del Oeste, así como en numerosos de las Grandes Praderas y del Medio Oeste, Estados que, por lo demás, llevaban las de perder en términos demográficos, lo que explicaría que fuera precisamente en el Senado en donde el Progresismo tuviera una mayor fuerza en Washington (a través de figuras como Robert La Follette, de Wisconsin) ${ }^{33}$.

\section{b) Marco procedimental}

El incremento del fenómeno filibusterista hará que se recrudezca el debate acerca de la libertad de palabra y la ausencia de límites de la misma en el Senado. Así, serán frecuentes las argumentaciones en favor y en contra de la misma en el curso de los debates. En favor de aquélla se aducirá, junto al argumento clásico que veía en el mismo un instrumento de defensa de las minorías, la consideración de que el filibusterismo impediría en muchas ocasiones la aprobación de medidas no apoyadas por la mayoría social (pues la mayoría senatorial puede no corresponderse con aquélla). Las posiciones contrarias destacarán que el mismo se opone a la necesaria conducción de la vida política del país, provocando el bloqueo frente a necesarios avances, llegándose a evocar las reformas que por la época estaban aprobando otros países con el fin de limitar la duración del debate.

Desde el punto de vista procedimental, se asiste a la articulación por vez primera de algunos remedios, por menores que fueran, contra el obstruccionismo, primeros intentos de encontrar una vacuna que se hallaría en 1917. Será en 1908, con ocasión

33 Véase, entre otros, MILKIS, M. et MILEUR, M. Progressivism and the New democracy. University of Massachussets Press, 1999; WALTER, N. Progressivism. A very short introduction. Oxford University Press, 2010. 
de la tramitación del conocido como Aldrich Bill y del intenso filibusterismo entablado frente al mismo, cuando se pongan en juego las primeras restricciones a la panoplia filibusterista. Así, como consecuencia de decisiones de la Presidencia del Senado, confirmadas en apelación por el Pleno, se adoptarían tres importantes novedades: a) En primer lugar, se decide que la presidencia pueda tener en cuenta a los senadores presentes, voten o no, a los efectos del cómputo del quórum de votación; b) Se acuerda que el mero debate no puede considerarse «bussiness» a efectos de permitir la petición de comprobación del quórum subsiguiente a otra solicitada en el mismo debate; c) Por último, se afirmó la posibilidad de que la mayoría del Senado, a través de su Presidente, hiciera más estricta la prohibición de intervenir más de dos veces en el mismo día. Como consecuencia de las novedades señaladas, desde la fecha indicada el medio obstruccionista más empleado será el de los discursos irrestrictos.

\section{c) Principales casos}

Como se indicara con anterioridad, el período analizado supuso el cénit del filibusterismo precloture (se ha hablado de filibusterismo irrestricto), incrementándose sustancialmente el número de proyectos obstruidos: así, en estos años se han computado 30 filibusterismos (más del doble que en la etapa precedente), de los que 16 fueron exitosos $(53 \%)^{34}$. Por otro lado, se amplían las «áreas temáticas» sobre las que opera el filibusterismo. El desarrollo acelerado del país y las tensiones que llevará consigo estarán detrás de muchos de los episodios obstruccionistas: así, el equilibrio entre territorios, la cuestión de la plata, o las crisis económico-financieras o «crisis de crecimiento» que periódicamente sobrevendrían. Junto a ello, el Sur bloqueará los tímidos intentos de reforma por mejorar la situación de la minoría afroamericana. Asimismo, aparecen ya los primeros filibusterismos en relación con nombramientos presidenciales.

El primer filibusterismo a destacar se inscribe en el último ámbito señalado, teniendo lugar en 1881 a propósito de determinados nombramientos realizados por el presidente saliente Hayes, siendo obstruidos por los propios republicanos mediante vanishing quorums. Una vez que tomó posesión la nueva administración del presidente Garfield serían los demócratas quienes bloqueasen otras medidas, consiguiendo que fueran retiradas las propuestas más polémicas de éste. En el mismo año tiene lugar un filibusterismo de carácter eminentemente interno, marcado por la incertidumbre en el equilibrio de fuerzas en el Senado. Se estaba esperando la designación de cuatro senadores por sus legislaturas estatales, lo que en principio habría de cambiar la mayoría, ante lo que los demócratas trataron de acelerar la elección de los miembros

34 BINDER, S. A. et SMITH, S. S. Politics or Principle?... op. cit. pp. 60 y 91. Con todo, como los propios autores reconocen, a los señalados habría que añadir otros supuestos en los que la obstrucción no fue tan «evidente» como en los casos referidos, articulándose por medios más camuflados (como las comprobaciones de quórums). 
de las Comisiones senatoriales, no obstante lo cual los republicanos consiguieron aguantar el tiempo necesario. La recién estrenada mayoría republicana impulsó la designación de nuevos Secretario y Sargento de Armas, medida que fue obstruida por los demócratas mediante discursos prolongados y mociones dilatorias ${ }^{35}$. Finalmente, la renuncia de dos senadores republicanos dio al traste con la nueva mayoría, habiendo conseguido el filibusterismo su objetivo tras haber consumido 26 días del calendario parlamentario.

Caso reseñable es el filibusterismo emprendido en 1890 contra un proyecto de ley de educación por estar protagonizado por un solo senador, quien además utilizó como táctica de presión el bloqueo de cualquier otra iniciativa (sin éxito finalmente). En ese mismo año comenzó el célebre filibusterismo frente al conocido como Force Bill (Federal Elections Bill), proyecto que pretendía hacer efectiva la vigencia en el Sur de la XIV Enmienda. Ante la férrea oposición sureña, los republicanos optaron por llevar al Senado a sesiones continuas con el fin de desgastar a aquella. Incluso se intentó introducir la moción de cierre por la vía de la hoy conocida como opción convencional. Finalmente, los demócratas consiguieron hacer fracasar el Force Bill gracias al apoyo de senadores silveritas ${ }^{36}$, con quienes se pactaron contrapartidas (el obstruccionismo había durado 33 sesiones) $)^{37}$.

En 1893 tuvo lugar un filibusterismo interpartidista con ocasión de la tramitación de un proyecto encaminado a suprimir las compras de plata. Las tácticas y contratácticas fueron las clásicas: intervenciones prolongadas (un senador «se extendió» por más de 200 páginas del Record) y sesiones continuas (la comenzada a las 11 de la mañana del 11 de octubre no se levantaría sino hasta las dos de la madrugada del día 13). Destaca también el empleo de quorum evanescentes por senadores presentes que se negaban a contestar a la comprobación, lo que dio lugar a un acalorado debate. El filibusterismo se extendió durante 46 días de sesión, aunque finalmente fracasó. Cuatro años más tarde sería un proyecto de financiación naval el

35 KOGER llega a computar la presentación de hasta 103 mociones dilatorias (Filibustering... op. cit.p. 73).

36 La reivindicación «silverista» o de acuñación de plata será característica del período. La reanudación de dicha acuñación, suspendida en 1873, fue demandada por los campesinos y por los Estados del Oeste (donde se habían descubierto nuevos yacimientos de dicho mineral) con el objetivo de crear una inflación favorable que incrementara el precio de unos productos agrícolas que habían experimentado importantes bajadas desde la década de 1870. El movimiento populista, primera versión de lo que más tarde sería el partido progresista, tendría dicha reclamación como una de sus señas de identidad, reivindicación también sustentada por los senadores demócratas del Sur y del Oeste. Los partidarios de que se acuñara moneda de plata obtendrían algunos éxitos parciales (así, la Ley Bland-Allison de 1878 o la Sherman Silver Purchase Act de 1890). Véase, entre otros, FOHLEN, C. La América anglosajona de 1815 a nuestros días. Labor, Barcelona, 1967, pp. 44-45; y BOSCH, A. Historia de Estados Unidos. Crítica, 2005, pp. 272-273.

37 WAWRO y SCHICKLER (Filibuster... op. cit. p. 121) estiman que antes de la aprobación del cloture en 1917 alrededor de un tercio de los filibusterismos se concentraron en los últimos días de cada Congreso o Legislatura. 
objeto de un filibusterismo reseñable, pues a lo largo del mismo se estableció el precedente de que había de mediar debate entre las distintas solicitudes de quórums.

Un filibusterismo peculiar se produjo en 1901 a propósito de un proyecto de ley sobre ríos y puertos, pues todo parece indicar que su verdadero impulsor era la Casa Blanca, quien empleó para ello a un solo senador, próximo ya al retiro. En este caso el filibusterismo operó como sustitutivo del veto presidencial (ya que la oposición republicana no podía manifestarse abiertamente en contra, dado que el proyecto era una contrapartida prometida en su día a la oposición demócrata). Dos años más tarde se produciría un episodio ilustrativo de la fuerza de la mera amenaza de obstrucción. En la noche del 3 de marzo, cuando faltaban horas para terminar el período de sesiones y estaban pendientes de aprobación importantes iniciativas, un senador por Carolina del Sur, deseoso de que se viera aprobado un proyecto de compensación a su Estado por los daños sufridos en la guerra de 1812, apiló libros desde el suelo hasta su pupitre (entre ellos los poemas de Byron), consiguiendo finalmente su objetivo ${ }^{38}$. En la misma sesión de 1903 tuvo lugar un interesante filibusterismo, ya que fue protagonizado (si bien no exclusivamente) por el presidente de una Comisión (la de Asuntos Territoriales, cuya mayoría respaldaba la posición de aquél), quien llegó a ausentarse de Washington a fin de impedir la votación, ya que existía la convención de que la misma no pudiera efectuarse en su ausencia (se trataba de la admisión de Arizona, Nuevo México y Oklahoma, siendo el punto de discordia la de este último).

El filibusterismo del que fuera objeto el denominado proyecto Aldrich es sin duda uno de los más célebres de la historia senatorial, asistiéndose a un auténtico «duelo al sol» entre dos grandes tácticos parlamentarios, el patrocinador del proyecto y líder de la mayoría republicana, Aldrich, y el progresista Robert La Follette, opuesto a un proyecto que pretendía paliar la crisis de 1907 introduciendo una mayor flexibilidad monetaria, aspecto juzgado como insuficiente por el último. En el curso del mismo (entablado contra el informe de la conferencia bicameral de conciliación) se consolidarían (en algún caso mediante aprobación por el Pleno) importantes precedentes en relación con los obstruccionismos: cómputo de los senadores presentes a efectos del quorum de votación, mediación de debate entre comprobaciones de quorum... Las sesiones estuvieron plagadas de incidentes ${ }^{39}$ : La Follette solicitó y obtuvo treinta comprobaciones de quorum en una única sesión; se expulsó a un asistente del mismo, presente permanentemente en el hemiciclo a fin de avisar ante cualquier momentánea falta de quorum (de cara a lograr la suspensión de la sesión); e incluso, en el transcurso de una afamada intervención de más de cinco horas (entre las dos y siete de la mañana), al beber el vaso con el combinado de leche y huevo procedente del restaurante con el que recargaba energías, el senador por Wisconsin sufrió una intoxicación (al parecer, el «preparado» lo era en un doble sentido) ${ }^{40}$. El

38 MACNEIL, N. et BAKER, R. A. The American Senate..., op. cit. p. 314.

39 Véase BURDETTE, F. Filibustering..., op. cit. pp. 83-90.

40 Véase UNGER, N. C. Fighting Bob La Follette: The Righteous Reformer. University of North Carolina Press, 2000, pp. 181-183.

(C) UNED. Revista de Derecho Politico

N. ${ }^{\circ} 111$, mayo-agosto 2021, págs. 137-162 
fin del filibusterismo no estuvo tampoco exento de gran controversia. Siendo relevado por dos senadores a quienes La Follette había cedido la palabra a fin de darse un respiro, uno de ellos, quien había leído extensos panfletos (entre ellos uno describiendo el sistema monetario de Portugal), cedió la palabra al otro, el cual era invidente, quien poco más tarde le devolvió el uso de la palabra cuando en realidad no estaba presente, pues había salido momentáneamente al vestíbulo. Aldrich no dejó escapar la ocasión y pidió la llamada a votación. Cuando la oposición fue consciente de la maniobra pidió la reconsideración presidencial, pero al haber comenzado la votación ya no podía interrumpirse.

En 1911 tuvo lugar un filibusterismo remarcable por el motivo que lo originó. Así, un senador se opuso a la admisión en la Unión de Nuevo México, dado que la misma no iba acompañada de la de Arizona, por cuanto que el presidente Taft recelaba de un Estado cuyo proyecto de Constitución acogía una muy amplia panoplia de instrumentos de democracia directa. Próxima la fatídica fecha del 4 de marzo, y ante un filibusterismo que obstaculizaba la aprobación de otros proyectos importantes, se llegó al compromiso de excepcionar por consentimiento unánime la regla que prohibía dos intervenciones en una misma sesión, a cambio de que abandonara el uso de la palabra para permitir la votación de otros bills. El filibusterismo fue finalmente exitoso.

La victoria de Wilson en las elecciones de 1912 dio lugar al comienzo de una serie de filibusterismos protagonizados por los republicanos, quienes trataron de frenar con ellos la agenda presidencial, particularmente activa. Así, en 1913 será la oposición al nombramiento de un nuevo Director del Censo (pues se consideraba que el anterior debía permanecer en su puesto hasta el fin del año fiscal) la que suscitaría una amenaza de obstrucción finalmente exitosa. De este modo, un senador apiló en su pupitre multitud de periódicos, algunos de los cuales databan de la guerra civil, mientras que otro anunció que podía relatar a la Cámara estadísticas desde la época del rey David. Un año más tarde sería un proyecto sobre financiación de ríos y puertos la ocasión de otro filibusterismo en el que se plantearía una cuestión de relevancia. Así, era tradicional que los senadores filibusteristas obtuvieran un respiro a lo largo de sus intervenciones mediante la cesión de la palabra a otro colega a fin de que éste le formulara pregunta, una formulación que iba a acompañada de largas exposiciones introductorias. Un senador planteó a la presidencia el impedir tales cesiones salvo consentimiento unánime de la Cámara, petición atendida por la presidencia temporal, confirmada en apelación por el Pleno. En cualquier caso, 24 horas más tarde, serenados los ánimos, un nuevo presidente pro tempore revocó tal criterio y volvió al anterior, decisión confirmada en apelación por el Pleno (el filibusterismo concreto fue exitoso) ${ }^{41}$.

En octubre de 1914 tuvo lugar otro caso interesante de filibusterismo, protagonizado por senadores demócratas sureños que pretendían forzar la aprobación de una ley de ayuda a los productores de algodón ${ }^{42}$. En tanto en cuanto en el sistema

41 BURDETTE, F. Filibustering..., op. cit. pp. 97-101.

42 BURDETTE, F. Filibustering..., op. cit. pp. 101-103. 
estadounidense una Cámara no puede suspender sus sesiones por más de tres días sin una resolución conjunta de ambas, se obstruyó la resolución del Senado, una vez aprobada la de la Cámara baja. Ésta apuró muchos los plazos y remitió su resolución con poco margen respecto a la fecha de comienzo de la pretendida suspensión. Los filibusteristas bloquearon la votación mediante múltiples peticiones de comprobaciones de quorum y presentaciones de mociones incidentales. Con todo, dada la proximidad de elecciones, muchos senadores se marcharon a sus circunscripciones, por lo que en el período «prorrogado» no había quorum de votación, ante lo cual los filibusteristas claudicaron.

Particularmente intenso, además de exitoso, fue el filibusterismo emprendido en 1915 contra el proyecto de compra pública de barcos mercantes (Ship Purchase Act) ${ }^{43}$. Los oradores republicanos se sucedieron hora tras hora, consumiendo semanas de trabajo parlamentario. La mayoría demócrata decidió entonces recurrir a la sesión continua, con múltiples incidentes e intervenciones. Un senador intervino durante más de once horas, llegándosele a denegar por la Presidencia la posibilidad de reposar sobre el brazo de su escaño. Posteriormente, ante la deserción de siete senadores demócratas que deseaban ver aprobadas otras iniciativas y la presentación de una solicitud de vuelta del proyecto a Comisión, la mayoría demócrata «filibustereó» dicha votación hasta recomponer la disciplina de partido. La sesión comenzada a mediodía del 8 de febrero no se levantó hasta las seis de la tarde del día 10, con los senadores durmiendo en el Capitolio (las crónicas destacan que algunos iban vestidos de noche en plena mañana e incluso sin corbata). Se entabló una intensa discusión acerca de las bondades y vicios del filibusterismo con ocasión de la presentación de una solicitud de cierre, rechazada por la presidencia. El proyecto se envió a la conferencia de conciliación y cuando volvió al pleno en la sesión del 3 de marzo, la inscripción como oradores de 23 senadores republicanos lo condenó definitivamente. El filibusterismo se había extendido durante 26 días y 702 páginas del Congressional Record. $^{44}$

El punto culminante de los filibusterismos en este período, y que daría pie a la posterior aprobación del cloture, fue el que tuvo lugar en marzo de 1917 contra el proyecto (impulsado por el presidente Wilson) por el que se armaba a los barcos mercantes, en el contexto de la difícil neutralidad estadounidense en vísperas de su implicación en la I Guerra Mundial ${ }^{45}$. Los republicanos aislacionistas (principalmente, senadores progresistas de Estados del Oeste y medio Oeste con importante población de origen alemán) emprendieron una acción concertada mediante la sucesión de pro-

43 El proyecto suscitó el rechazo de determinados sectores por lo que suponía de intervencionismo público y, especialmente, por el temor de que la compra de barcos germanos, que se habían quedado «varados» en puertos estadounidenses tras el estallido de la Guerra Mundial, proporcionara una inyección económica sustancial para Alemania y su esfuerzo bélico.

44 Véase, KOGER, G. Filibustering... op. cit. pp. 149-151.

45 Un extenso relato de este filibusterismo en RYLEY, T. W. A Little Group of Willful Men: a Study of Congressional/Presidential Authority. Port Washington, N. Y.: Kennikat Press, 1975. 
longados turnos de palabra. Ante la situación, se presentaron reiteradas solicitudes de consentimiento unánime para fijar una hora para la votación, propuestas saldadas todas en fracaso. La enojada mayoría optó entonces por mantener al Senado en sesión continua. La situación se hizo más extraña cuando a la una y media de la mañana del día 3 de marzo llegó al Pleno el informe de la conferencia mixta de conciliación de un proyecto de financiación agrícola, que gozaba de tramitación preferente, lo que dio lugar a que algunos republicanos aprovecharan la ocasión mediante prolongadas intervenciones, para retrasar al máximo la vuelta a la consideración del Arm Ship Bill. A las tres y veinte de la madrugada un senador leyó un manifiesto firmado por 75 senadores anunciando que en caso de someterse a votación el proyecto sobre la marina mercante darían su voto afirmativo. Conforme avanzaba el reloj, los demócratas adoptaron una nueva estrategia: impedir las intervenciones de los republicanos, evitando la extraordinaria publicidad alcanzada. Así, la mayoría acaparó el uso de la palabra o, lo que es igual, «filibustereó» el filibusterismo de la minoría. Finalmente, alcanzado el mediodía del día 4 de marzo, la sesión se suspendió sine die. Los obstruccionistas habían conseguido su objetivo.

Las reacciones no tardaron en producirse, en un contexto de gran tensión ante la aventurada como inminente guerra con Alemania. La más célebre, la del Presidente Wilson. «Un pequeño grupo de hombres caprichosos» («willful men») había secuestrado al país. En palabras del mandatario, «el Senado de Estados Unidos es el único cuerpo legislativo del mundo que no puede actuar cuando la mayoría está lista para la acción». El único remedio frente a ello, añadía el dirigente, pasaba por modificar las normas del Senado. Se identificó a once senadores que habían liderado y protagonizado el filibusterismo, sobre los cuales recayó buena parte de la ira política e incluso popular. Así, se sucedieron las declaraciones e incluso las resoluciones de legislaturas estatales (entre otras, el Senado de Kentucky o la Cámara de Tennessee) condenando a los mismos por antipatriotas ${ }^{46}$. Nunca antes el filibusterismo había concitado tanta tensión y, también, por ende, tanto rechazo.

\section{LA APROBACIÓN DEL CLOTURE. CONCLUSIONES}

En marzo de 1917, tras el bloqueo de los proyectos wilsonianos ya referidos, se convocó una sesión especial en la que, sorprendentemente sin mucho debate, se procedió a aprobar con amplio apoyo (76 votos a favor y sólo 3 en contra) la reforma de la Regla XXII. Por la misma se introducía la moción de cierre, posibilitándose su

46 Un grupo de ciudadanos de Mississipi envió al senador Vardaman una gran cruz de hierro con la siguiente inscripción: «Por si al Kaiser se le olvida». 
presentación por un mínimo de 16 senadores, y cuya aprobación requería el voto favorable de las dos terceras partes de los senadores presentes ${ }^{47} .{ }^{48}$

Se ha llegado a señalar ${ }^{49}$ que ya incluso desde el mismo momento de su adopción, la mayoría de los senadores no pensaban que la mencionada reforma fuera a suponer el fin del obstruccionismo (y mucho menos, cabría añadir, el fin de la tradicional regla de debate ilimitado en el Senado). De hecho, la mera invocación o presentación del cloture fue muy escasa en las primeras décadas de su existencia. Así, entre 1917 y 1927 únicamente se votaron diez mociones de cierre, de las cuales sólo cuatro fueron aprobadas. La primera de ellas se produjo a raíz del debate sobre la ratificación del Tratado de Versalles en 1919, acordándose por una amplia mayoría (republicanos y demócratas coincidían en someter a votación el mismo, aunque por razones opuestas). La segunda ocasión en que fuera acordado el cloture también fue a propósito de la ratificación de un tratado, en este caso, el Protocolo sobre el Tribunal Internacional en 1926, y un año más tarde se aprobaría respecto a dos proyectos: uno bancario y

47 En concreto, la recién aprobada norma establecía lo siguiente: «Si en cualquier momento una moción firmada por 16 senadores con el objeto de poner fin al debate una cuestión pendiente es presentada al Senado, el Presidente deberá plantear de inmediato la moción, y en el segundo día siguiente de trabajo, una hora después de que el Senado se haya reunido, deberá sustanciar la moción ante el Senado y ordenar al secretario llamar a votación, y tras comprobar que existe quorum, el Presidente deberá, sin debate, someter a votación de «síes» o «noes» la cuestión: ¿¿Es la opinión del Senado que el debate debe ser llevado a su fin?'. Y si la cuestión es decidida en sentido afirmativo por el voto de dos terceras partes de los senadores «votantes», entonces la medida será considerada asunto no finalizado, excluyendo cualquier otro asunto hasta baya sido resuelto. A partir de entonces ningún senador estará facultado para intervenir más de una hora en total en relación con el asunto pendiente, incluidas las enmiendas, así como las mociones relativas a la misma, y será deber del Presidente velar para que se cumplan estos límites. Salvo por consentimiento unánime, ninguna enmienda podrá sustanciarse después de la votación sobre el cierre del debate, a menos que la misma bubiera sido presentada y leída con anterioridad a dicho momento. Ninguna moción dilatoria, enmienda de igual carácter, o enmienda no bomogénea (germane) podrá tramitarse. Las cuestiones de orden, incluidas las relativas a la relevancia, así como las apelaciones a decisiones de la presidencia, serán decididas sin debate previo.».

48 En 1949 y 1959 se aprobarían sendas modificaciones de la Regla XXII. En la primera fecha indicada se acordó la extensión del cierre del debate a las denominadas mociones para proceder (ventana abierta por la que se "colaban" numerosos filibusterismos), con excepción de las atinentes a iniciativas de reforma de las propias reglas del Senado (estas se blindaban con una protección extra). A cambio de esta mayor amplitud del cloture, se introdujo la novedad de que el mismo requería para su aprobación el voto favorable de las dos terceras partes de los miembros de derecho del Senado. La siguiente reforma tuvo lugar en 1959, siendo fruto en buena parte de un intenso debate producido en los años precedentes en el que se planteó incluso la posteriormente conocida como opción nuclear o constitucional, esto es, la posibilidad de que el Senado, a comienzos de cada renovación parcial, pudiera aprobar desde cero sus propias reglas de procedimiento. Como alternativa a esta radical medida, finalmente, en 1959 se alcanza un compromiso por el que se modifica la Regla XXII rebajándose la mayoría requerida para el cloture a dos tercios de los senadores presentes y votantes y, de otra parte, se amplía su ámbito para incluir a las mociones para considerar cambios en las Reglas del Senado. Posteriormente, mediante reforma de 1975 la mayoría exigida se rebajaría a la actual de 3/5 de los miembros de derecho del Senado.

49 BURDETTE, F. Filibustering in the Senate, op. cit., p. 128.

(C) UNED. Revista de Derecho Politico

N. ${ }^{\circ} 111$, mayo-agosto 2021, págs. 137-162 
otro relativo a la «Prohibición». Entre 1928 y la promulgación de la Ley de Derechos Civiles de 1964 el cierre del debate solo se aprobaría en dos ocasiones (para esta última y en la tramitación del proyecto de ley de comunicaciones por satélite en 1962). Habría que esperar a los años sesenta para asistir a su utilización clave para la aprobación de la legislación que pusiera fin a la discriminación racial en el Sur, dando paso a una nueva época.

La aprobación de la regla del cierre del debate en el Senado estadounidense en 1917 no supuso, pues, un cambio radical en la situación imperante con anterioridad. Cierto es que, por vez primera, se establece un límite o cortapisa a la libertad irrestricta de palabra o debate, considerado un pilar intocable del Senado; sin embargo, la operatividad de la medida sería prácticamente nula. En primer término, por el propio tenor de la Regla XXII, ya que se dejaba la puerta abierta al filibusterismo en relación con las mociones para proceder o considerar una iniciativa (una suerte de toma en consideración previa a todo debate plenario), por cuanto que para las mismas no se preveía el cierre del debate. Por otra parte, y, sobre todo, por cuanto que no existía una auténtica voluntad política de erradicar el filibusterismo.

Mientras que otros países por la misma época ya habían puesto freno al obstruccionismo parlante mediante la articulación de un efectivo cierre del debate o de otros mecanismos similares como la guillotina o el canguro ${ }^{50}$, en el caso del Senado estadounidense el filibusterismo pervivió como decisión consciente de la mayoría de sus integrantes (y aún hoy). De hecho, los senadores norteamericanos fueron bien conscientes de que aquel era un instrumento insustituible que reforzaba su posición individual y, por tanto, su importancia en el conjunto del arquitrabe político washingtoniano. Además de esa autoconciencia de la relevancia del propio papel (y de uno de los resortes más importantes en ello), el filibusterismo era valorado como instrumento de defensa del grupo minoritario en el Senado frente a la mayoría, siendo muy consciente la mayoría de hoy de que en un breve lapso (dos años) podía pasar a ser minoría, interesándole contar con tal instrumento. Por otra parte, el filibusterismo operaba como instrumento de negociación para la defensa de los intereses de los Estados con menor peso en la Unión, sin olvidar tampoco que, en su aspecto más siniestro, actuó (sobre todo en el siguiente período) como elemento de bloqueo por los representantes sureños de cualquier intento por poner fin a la segregación racial en sus Estados ${ }^{51}$.

50 Así, cabe recordar, entre otros casos, la aprobación en 1881 por la Cámara de Comunes británica del cloture by compartments (por el que articulaban límites temporales máximos de debate en cada una de las fases de tramitación de un proyecto) o la reforma del Reglamento del Congreso de los Diputados español en 1918 por la que se establece un mecanismo similar.

51 BINDER y SMITH (Politics or Principle?... op. cit. pp. 85-86) señalan al respecto que, si bien los temas conectados con los derechos civiles (expansión hacia el Oeste, derechos de voto...) ha sido la materia «individual» más obstruida en el conjunto de filibusterismo hasta 1992, el número de proyectos «filibustereados» concernientes a otras materias ha sido muy superior ( 45 medidas relativas a derechos civiles frente a un total de 289 medias obstruidas, lo que significa un $15 \%$ del total). Por períodos, cabe destacar que los derechos civiles fue la materia dominante en dos etapas: la inmediatamente ante- 
Title

Precloture filibuster in the U.S. Senate (1789-1917)

\title{
Summary
}

I. INTRODUCTION: THE CONCEPTUAL AND LEGAL GROUNDS OF THE CLASSIC FILIBUSTER. II. THE PRECLOTURE FILIBUSTER: PERIODS, PROCEDIMENTAL FRAMEWORK AND PRINCIPAL CASES. 1. The primitive Senate 1789-1820. 2. The filibuster between 1820 and 1880.3 . The cenit of the precloture filibuster: 1880-1917. III. CLOTURE ADOPTION. CONCLUSIONS

\section{Resumen}

El filibusterismo, modalidad estadounidense del obstruccionismo parlamentario, ha jugado y juega un papel capital en el sistema político norteamericano, siendo un elemento identificador del Senado y explicativo en buena parte de la trascendencia de éste. Plasmado esencialmente en la táctica de los discursos irrestrictos, basados en el principio de libertad de palabra, históricamente también tuvo otros mecanismos de plasmación tales como las reiteradas comprobaciones de quorum o los llamados quorum evanescentes. El artículo analiza la evolución del filibusterismo en la Cámara Alta estadounidense hasta la aprobación del cloture o cierre del debate en 1917, distinguiéndose tres períodos: el del Senado primitivo (17891820), en el que únicamente hay fenómenos muy esporádicos que solo difícilmente pueden ser calificados como de filibusterismos stricto sensu; el del Senado clásico (1820-1880), marcado por las tensiones que conducirían al conflicto civil y las inmediatas consecuencias del mismo; y el del auge del filibusterismo (1880-1917), en el que el fenómeno se multiplica exponencialmente hasta dar lugar a la reacción de 1917 con la aprobación de la Regla XXII, que estableció la posibilidad de cerrar el debate si así lo aprobaban dos tercios de los senadores presentes. No obstante, la aprobación de la referida Regla no supuso el fin del filibusterismo, siendo rara vez empleado el cierre del debate en el período que transcurre hasta la década de los sesenta. Así, las peculiaridades del Senado norteamericano y del rol jugado por sus integrantes, en contraste con lo sucedido en otros parlamentos del mundo, explicarían su supervivencia hasta nuestros días.

\begin{abstract}
The filibuster, as is known the parliamentarian obstruction in the United States, has played, and still plays, a key role in the US political system, being an identifying element of the Senate and explains the relevancy of this Chamber. The filibuster is identified with the tactics of unlimited
\end{abstract}

rior a la Guerra de Secesión (1837-1861) y en el período entre 1937 y 1967, no siendo así entre 1880 y 1936 y a partir de 1967.

(C) UNED. Revista de Derecho Politico

N. ${ }^{\circ} 111$, mayo-agosto 2021, págs. 137-162 
speeches, but historically it has also employed other tactics as the multiple quorum calls motions and the so-called vanishing quorums. This article analyses the evolution of the filibuster in the historic Senate until the approval of the first filibuster antidote, the cloture rule in 1917. Three periods can be distinguished: the primitive Senate, until 1820, where there is not a real filibuster; the classic Senate, until 1880, characterized by the tensions which led to the civil war and its aftermath; and the golden age of the filibuster precloture until the passing of the Rule 22, which established the possibility of ending the debate with the approval of $2 / 3$ of the voting senators. Nevertheless, the cloture didn't imply the filibuster deletion, as the cloture was rarely invoked until de sixties. The Senate particularism and the role played by senators into the American checks and balances would explain its survival until our days, in contrast with what has happened in other parliaments.

\section{Palabras clave}

Filibusterismo; obstruccionismo; Senado; libertad de palabra; quorum; cloture.

\section{Key words}

Filibuster; obstructionism; Senate; freedom for speech; quorum calls; cloture. 